\title{
Multi-Radix Trivium Implementations for Low-Power IoT Hardware
}

\author{
J. M. Mora-Gutiérrez, C.J. Jiménez-Fernández, M. Valencia-Barrero
}

\begin{abstract}
The integration of lightweight symmetric encryption is becoming increasingly widespread in very low power Internet of Things (IoT) applications, with the rapid emergence of very low energy block and stream ciphers in portable and wireless systems. Trivium is one of the lightweight stream ciphers shortlisted for the hardware profile of the eSTREAM project in 2008. This paper describes low-power multi-radix Trivium implementations based on the use of parallelization techniques to reduce dynamic power consumption. The low power Trivium designs were implemented and characterized in TSMC $90 \mathrm{~nm}$ to compare area resources and power reduction. The implementation results show that our proposed designs offer dynamic power savings of $31-45 \%$ with radix-1 and radix-2 when compared with the current Trivium, and $15 \%$ with radix-8. There is no improvement, however, with radix-16.
\end{abstract}

Index Terms-Lightweight cryptography, stream ciphers, Trivium, low-power, ASIC implementation, IoT hardware.

\section{INTRODUCTION}

$\mathrm{P}$ rivacy, data protection and information security are key requirements for IoT hardware applications. However, many of the communications tasks that take place in IoT applications are between battery-based portable devices with limited computing resources, in which the mechanisms of encryption and authentication are severely constrained.

Logically, therefore, one of the main priorities in IoT designs is to choose lightweight cryptographic algorithms with low power consumption (lightweight cryptography) $[1,2]$ and apply techniques that reduce dynamic power consumption [3]. Stream ciphers like Grain, Mickey and Trivium, featuring algorithms specially designed for optimal performance in hardware cryptography, were identified and published in the European initiative known as the eSTREAM (ECRYPT Stream Cipher) Project [4-6]. These algorithms are now being used in battery powered devices, passively-powered systems and healthcare instruments with wireless communication devices [6-12], where power consumption is an important design criteria.

We selected the Trivium stream cipher as a low-resourceconsumption cryptographic algorithm very well suited to this type of systems. From the theoretical perspective of design techniques for power reduction, this work focuses on improving dynamic consumption at a logical level. As will be explained in the next section, the parallelization technique [1315] is a good option for reducing power consumption in Trivium because this cipher's internal architecture, based on shift registers, lends itself well to parallelization.

Two different parallelization methods were proposed [16] for radix-1. Firstly, the mixed parallel low power (MPLP) option, where parallelization is applied to flip-flops unaffected by non-linear feedback paths; and secondly, the full parallel low power (FPLP) option, where parallelization is applied to all the flip-flops in the Trivium cipher even though this implies redesigning non-linear feedback paths.

This paper focuses on multi-radix Trivium hardware implementations for low power IoT applications. The objective is to propose an ASIC implementation of low power multi-radix Trivium designs based on standard cell libraries in CMOS technologies, in order to compare area resources and power consumption.

To compare the benefits of each of the proposed solutions, twelve versions of Trivium (four for each standard, MPLP and FPLP versions) were designed in a 90-nanometer technology to obtain place-and-route parasitic parameters from the layout level, as shown in Fig. 1. For this purpose, a detailed study of dynamic power consumption was carried out, extracting full timing and post-layout parasitic parameters. The results show that the application of this technique makes it possible to reduce dynamic power, decreasing power consumption by up to $45 \%$ (depending on the multi-radix option used), with no performance loss and only a slight penalty in area.

The contents of the paper are organized as follows. Section II briefly describes the multi-radix Trivium algorithm and its hardware implementation. In section III, two low power multiradix architectures are presented along with power reduction results for a $90 \mathrm{~nm}$ CMOS technology process. Section IV compares the results obtained for the MPLP and FPLP Trivium options, and, finally, some conclusions are presented in Section V.
This work was partially funded by the following projects: CITIES, MCINN (TEC2010-16870/MIC) and CESAR (TEC2013-45523-R).

The authors are with the Instituto de Microelectrónica de Sevilla (IMSECNM), CSIC-University of Seville, 41092, Spain (email: jmiguel@imsecnm.csic.es, cjesus@imse-cnm.csic.es, manolov@dte.us.es). 


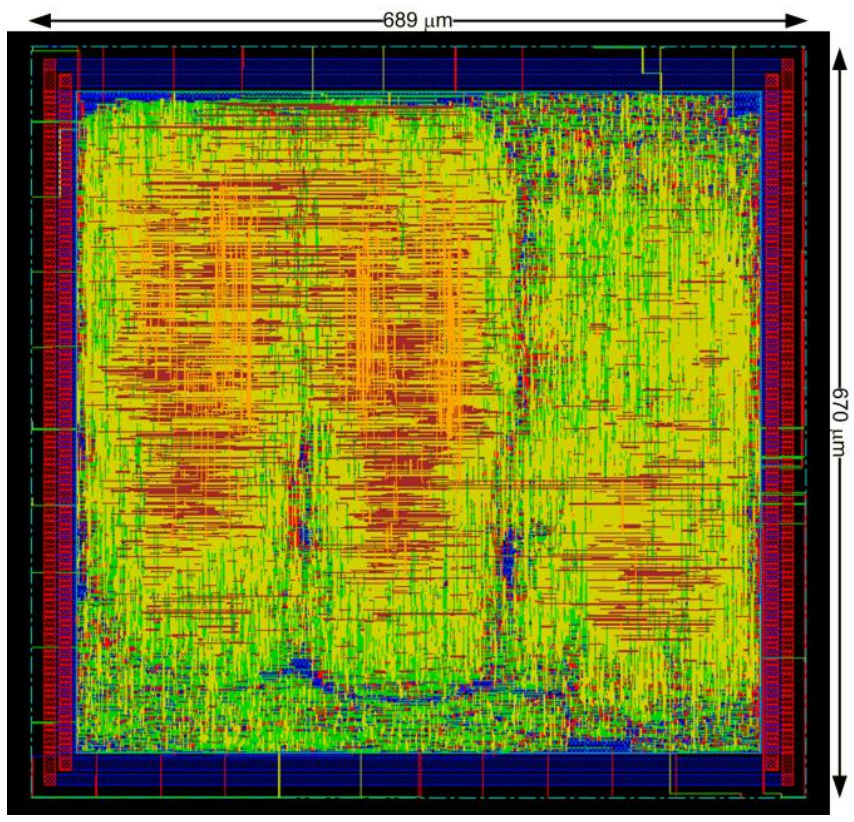

Fig. 1. Multi-Radix Trivium in a 90nm CMOS technology.

\section{STANDARD MUlTi-RADIX TRIVIUM HaRdWARE IMPLEMENTATION}

Trivium is a synchronous stream cipher designed to generate up to $2^{64}$ bits of pseudorandom key stream from an 80-bit secret key $(K E Y)$ and an 80-bit initialization vector $(I V)$. It was first proposed by Christophe De Canniere and Bart Preneel in 2006 [19].

The cipher's architecture is based on a 288-bit cyclic shift register (also called an internal state register), with combinational logic (AND and XOR gates) providing its nonlinear feedback. Implementations of the Trivium algorithm comprise three circular shift registers of different lengths. The output bits generated per clock cycle are called radix. This paper describes implementations of the Trivium algorithm that generate radix-1, radix-2, radix- 8 and radix-16. These multiple radices are generated using the same internal state register but shifting it one or more bits to the right depending on the radix $(1,2,8$ or 16 bits), as shown in the schematic representation of the radix- $m$ Trivium stream cipher in Fig. 2.
The length of each internal shift register is not the same; the first register has 93 bits, the second 83 bits and the third 111 bits. Each register is shifted serially with the inclusion of $m$ bits depending on the number of output bits, $m$, with $n=m-1$. Following is the VHDL code for loading each internal shift register (state):

- First shift register: state(92 downto 0) $<=\operatorname{state}((90-n)$ downto 0$) \& t 3($ n downto 0$)$;

- Second shift register: state(176 downto 93) <= state((174-n) downto (92-n)) \& t1 ( $n$ downto 0$)$;

- Third shift register: state $(287$ downto 177$)<=$ state $((285-n)$ downto $(176-n)) \& t 2(n$ downto 0$)$;

Signals $\mathrm{t} 1, \mathrm{t} 2$ and $\mathrm{t} 3$, are generated by logical operations of some state register bits and signals k1, k2 and k3. The VHDL code that produces these bits is given using the for...generate statement:

\section{G1: for $i$ in 0 to $n$ generate}

$t 1(i)<=k 1(i)$ XOR ((state 1 (90-i) AND state 1(91-i)) XOR

statel $(70-i))$;

end generate $G 4$;

G2: for $i$ in 0 to $n$ generate

t2(i) $<=k 2(i)$ XOR $(($ statel $(174-i)$ AND statel $(175-i))$

XOR state1 (263-i));

end generate $G 5$;

G3: for $i$ in 0 to $n$ generate

t3(i) $<=k 3(i)$ XOR ((state1(285-i) AND state1(286-i))

XOR statel $(68-i))$;

end generate 66 ;

G4: for $i$ in 0 to $n$ generate

$k 1(i)<=$ statel $(65-i)$ XOR statel $(92-i)$;

end generate G1;

G5: for $i$ in 0 to $n$ generate

$k 2(i)<=\operatorname{state1}(161-i)$ XOR statel $(176-i)$;

end generate $G 2$;

G6: for $i$ in 0 to $n$ generate

$k 3(i)<=\operatorname{statel}(241-i)$ XOR statel $(286-i)$;

end generate G3;

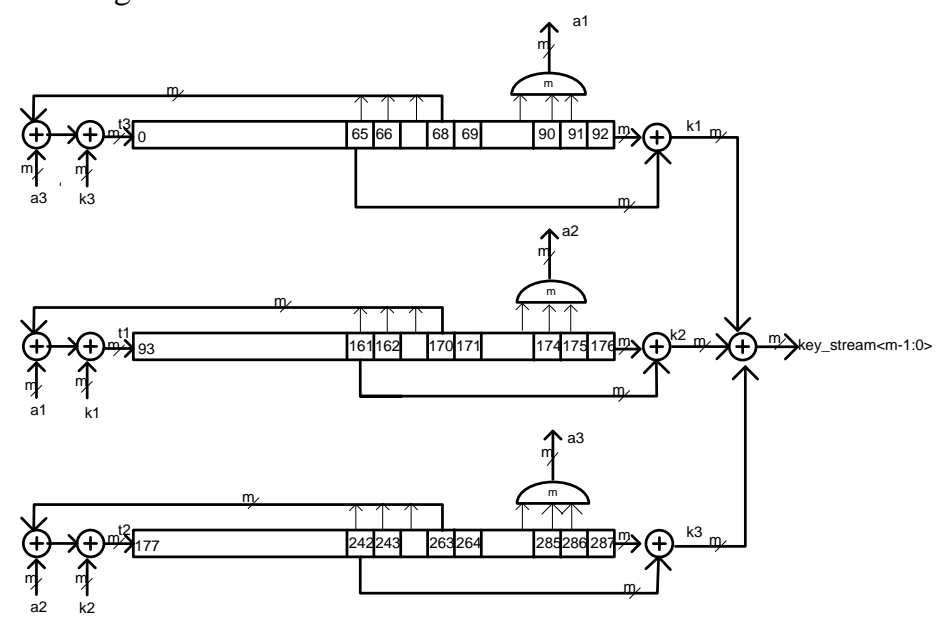

Fig. 2. Schematic representation of the radix-m Trivium cipher. 
The main Trivium process involves two phases: initialization and the updating of the states to generate keystream bits. First, the internal states are initialized, with the parallel loading of an 80-bit secret key (KEY) and an 80-bit initialization vector (IV), the remainder being zeros except for the last three, which are ones. Once loaded, the state register is shifted $4 \times 288$ clock cycles before a valid key stream can be obtained.

Following is the VHDL code for initialization process, which requires one clock cycle:

- First shift register: state(92 downto 0$)<=X^{\prime \prime} 0^{\prime \prime} \quad \&$ $K E Y$;

- Second shift register: state(176 downto 93) $<=X^{\prime \prime} 0^{\prime \prime}$ \& IV;

- Third shift register: state(287 downto 177) <= "111" $\& X^{\prime \prime} 0^{\prime \prime}$;

Multi-radix key stream bits are then generated every clock cycle. The bits to be introduced in Trivium's shift registers are generated by XOR gates. The VHDL code that produces these bits is given using the for...generate statement, being $n$ the radix minus one. The output key stream is an XOR of signals k1(i), k2(i) and k3(i). The VHDL code describing the operation is given by:

\section{G7: for $i$ in 0 to $n$ generate \\ key_stream $(i)<=(k 1(i)$ XOR $k 2(i)$ XOR $k 3(i))$; end generate $G 7$;}

With this architecture and mode of operation most of the power consumption is attributable to the flip-flops in the cyclic shift registers.

To reduce dynamic power consumption, we therefore focused on decreasing the switching activity by applying parallelization techniques, maintaining the same input frequency and supply voltage.

\section{A. Reducing Power consumption through Parallelization}

Power dissipation in digital circuits and systems is an important factor in the performance metrics of microelectronic circuits. Current renewed interest in low power consumption is a response to the decreasing size of technology processes, to higher clock frequencies and to the growing number of mobile, battery-operated systems.

In digital CMOS designs, the total power consumed by a circuit falls into two major categories: static power, which is the power consumed when the transistors are not switching, and dynamic power, which represents the power consumed by switching logic states and is divided into two components, switching power and internal power [17-18].

Dynamic power in clocked circuits therefore stems from the current that flows only when the device's transistors are switching from one logic state to another. This current flows mainly to charge and discharge parasitic capacitances (from gate and drain) and wire capacitances. In most electronic devices, dynamic power consumption [20-21] can be expressed as:

$$
P(\text { dyn }) \sim C l o a d, a, f, V d d^{2}
$$

As can be seen in equation (1), dynamic power consumption depends directly on switching activity factor $(a)$, which represents the average fraction of clock cycles in which a signal transition occurs, clock frequency $(f)$, supply voltage $(V d d)$ and output capacitance (Cload). The greater the logic transitions in the cell output, the greater the switching power.

In our study, dynamic power in shift registers was reduced by decreasing switching activity. This was done by applying the shift register parallelization technique, while maintaining the same frequency and supply voltage.

Dynamic power was analyzed with Synopsys tools using a switching activity file in SAIF (switching activity interchange format) and capacitances and power models for wires and cells taken from the technology library. This SAIF file was generated with logic timing simulations and a clock frequency of $25 \mathrm{MHz}$

Synopsys reports dynamic power as switching power and internal power. Switching power, or external power consumption, follows expression (1), where the external capacitance is computed from the pin capacitances specified in the library cells and net capacitances are calculated from the net-load nodes. Internal power includes the power consumed by the switching of the cells' internal nodes.

The Synopsys power estimator sums up these two circuit contributions in expression (2), where index $m=1 \ldots, \mathrm{M}$ refers to the cells instantiated in the circuit and $n=1 \ldots \mathrm{N}$ to the nets.

$$
P(d y n)=\sum_{m=1}^{M} \operatorname{Pint}_{m}+\sum_{n=1}^{N} \operatorname{Pswt}_{n}
$$

Dynamic power reduction is caused by a reduction in the switching activity in nodes because, even though the operating frequency remains the same, switching activity inside the flipflops is halved.

The Trivium implementations were described in $V H D L$ code, synthesized with Design Vision (Synopsys), and verified using the ModelSim (Mentor Graphics) simulation environment, with the same test vectors and using the same key and IV as those presented in the Trivium reference files [5].

\section{LOW POWER MULTI-RADIX TRIVIUM HARDWARE IMPLEMENTATION}

The parallelization technique cannot be applied directly to all the state register flip-flops in the Trivium stream cipher because the outputs of some of them are involved in combinational operations.

We propose two new low power multi-radix Trivium implementations using logic parallelization techniques: MPLP and FPLP. In MPLP, parallelization is applied to flip-flops unaffected by non-linear feedback paths, i.e., the less significant bits from each shift register; 196 out of 288 bits in the state register for radix-1 and radix-2, 144 out of 288 for radix-8 and 96 out of 288 for radix- 16 . 
Parallelization requires a slight hardware modification in each shift register shown in Fig. 2.

A schematic representation of the radix-2 MPLP Trivium is shown in Fig. 3. The first shift register contains bits 0 to 63; the second, bits 93 to 160 ; and the third, bits 177 to 240 . With the parallelization technique, the bits in each shift register not involved in feedback or combinational operations (LSB bits) are divided into two separate shift registers denominated odd and even shift registers. Each of these has half-bits, so the total length of the shift register remains the same.

In multi-radix MPLP Trivium, 1, 2, 8 or 16 bits are shifted to the right in each cycle, depending on the radix. The required modification involves generating a half frequency clock and adding a multiplexer in the least significant bit of each shift register.

The $K E Y$ and $I V$ are loaded in parallel, the even registers being loaded with the rising edge and the odd registers with the falling edge of a clock with half the frequency of the external clock.

If the parallelization technique were applied to all the Trivium stream cipher state register flip-flops, the reduction in power consumption would be bigger, because that is where most of the power consumption is dissipated. To achieve this, a second low power implementation, named full parallel low power (FPLP) Trivium, was designed. In this implementation, all the bits in the Trivium state register were parallelized. This required further modification to the standard version. Each Trivium shift register in Fig. 1 was split into two half-length shift registers denominated odd and even.

Fig. 4 shows a schematic representation of the radix-2 FPLP Trivium. The length of each shift register is indicated in the figure inside the odd and even registers. In radix-2 Trivium, two bits are shifted to the right in each cycle.

As mentioned in Section II, generation of the input bits in each of the shift registers and the key stream depends on the bits stored in different positions in the shift registers. It is therefore necessary to introduce extra logic to select the bits from the correct shift register, depending on whether the clock cycle was odd or even. On one clock edge, the bit to be retrieved will be in the even register and on the following clock edge, the bit to be retrieved will be in the odd register.

This implies the use of multiplexers which, using the clock as the selection signal, select the bit to be retrieved from the corresponding shift register, as shown in Fig. 4.

In the first clock cycles, both the secret key and the $I V$ are loaded in parallel, as described above for the MPLP implementation.

\section{A. Area and cell implementation report}

The area report provided by the Design Vision synthesis tool for the standard, MPLP and FPLP multi-radix Triviums is shown in Table I, which details the area occupied and the number of logical cells in each of the Trivium ciphers designed after the synthesis. The table also shows the areas of sequential (non-combinational) logic and combinational logic. The increments (\%) in each measurement compared to the corresponding 1-bit Trivium version are shown in Table II. To better appreciate the details of the synthesis tool's report, the data is also represented graphically in Fig. 5.

The results show that the resources consumed increase significantly as the number of output bits rises. For example, the increase in cells in the standard 16-bit version was 37\% higher than in the 1-bit version (see Table II). Something similar occurred in the MPLP version (with an increase of $47 \%$ ) and the FPLP version (with an increase of $85 \%$ ). Similar increases also occurred, although to a lesser extent, in the total area $(17 \%$ in the standard version, $16 \%$ in MPLP and $33 \%$ in FPLP).

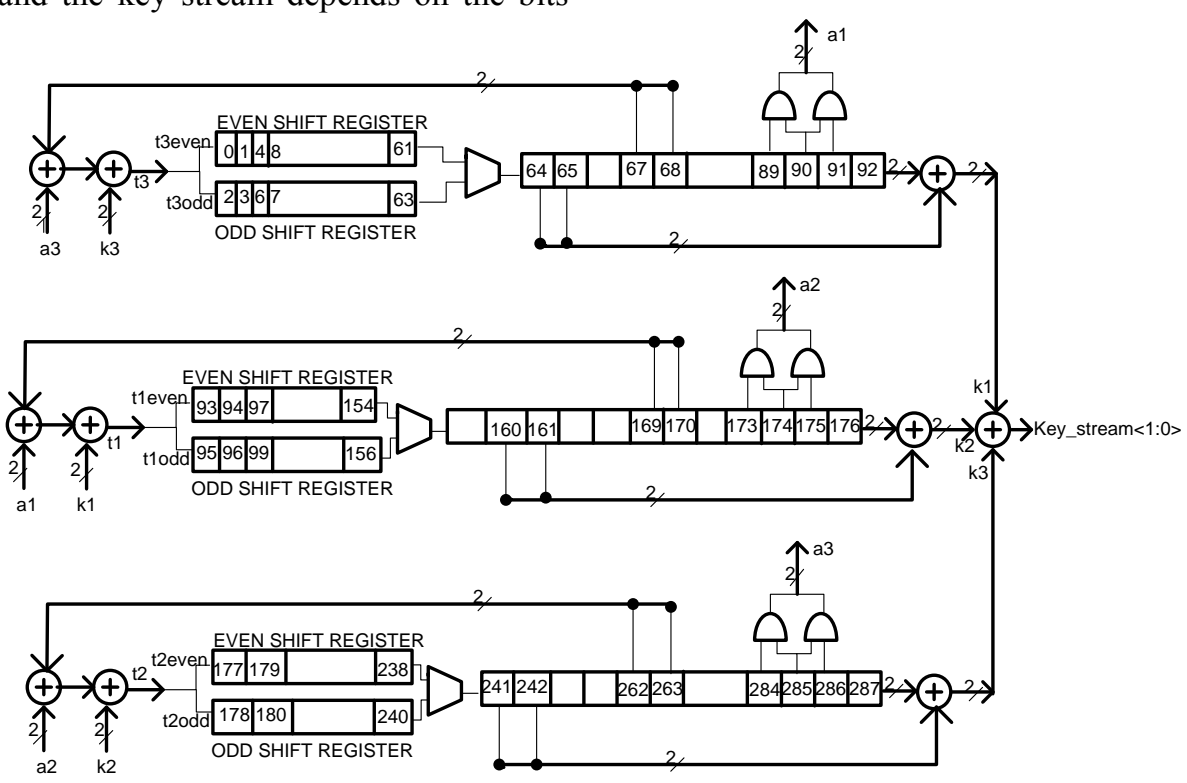

Fig. 3. Mixed-Parallel Low Power Trivium (MPLP) schematic, radix-2. 


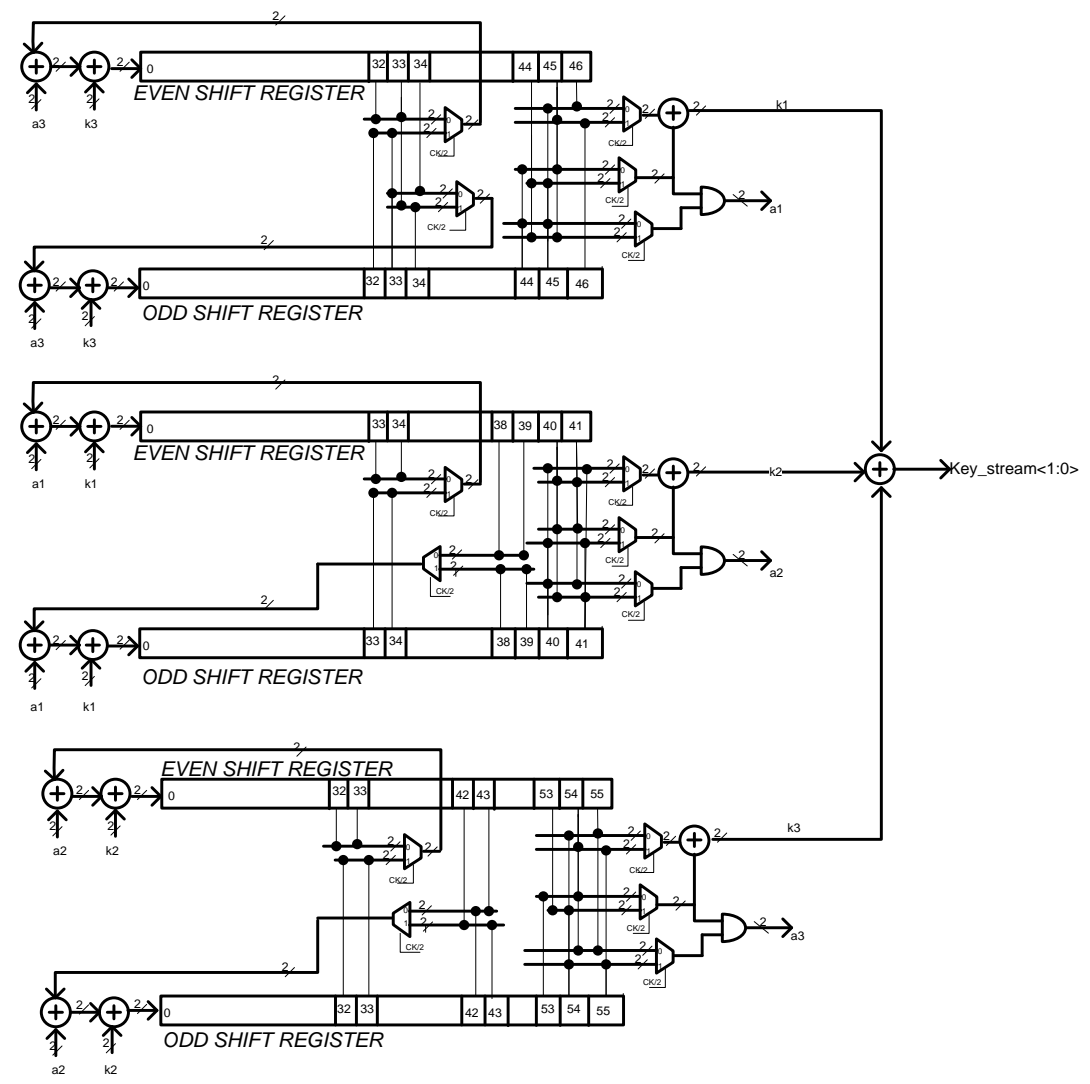

Fig. 4. Full-Parallel Low Power Trivium (FPLP) schematic, radix-2.

TABLE I

MULTI-RADIX TRIVIUM POST-SYNTHESIS REPORT.

\begin{tabular}{|c|c|c|c|c|}
\hline \multirow[b]{2}{*}{ Trivium } & \multirow[b]{2}{*}{$\begin{array}{c}\text { Total } \\
\text { Area } \\
\left(\mu m^{2}\right)\end{array}$} & \multicolumn{3}{|c|}{ Library: TSMC 90 nm } \\
\hline & & $\begin{array}{c}\text { Number } \\
\text { Logic } \\
\text { Cells }\end{array}$ & $\begin{array}{c}\text { Area Non } \\
\text { Comb. Cells } \\
\mu^{2}\end{array}$ & $\begin{array}{c}\text { Area } \\
\text { Comb. Cells } \\
\mu_{\text {. }}^{2}\end{array}$ \\
\hline Trivium_x1 & 6360.94 & 625 & 4390 & 1971 \\
\hline Trivium_xImp & 6603.00 & 665 & 4474 & 2128 \\
\hline Trivium_xlfp & 6750.47 & 620 & 4633 & 2117 \\
\hline Trivium_x2 & 6428.72 & 631 & 4387 & 2041 \\
\hline Trivium_x2mp & 6646.04 & 646 & 4545 & 2101 \\
\hline Trivium_x2fp & 6898.65 & 656 & 4629 & 2269 \\
\hline Trivium_x8 & 6851.37 & 723 & 4368 & 2484 \\
\hline Trivium_x8mp & 7070.81 & 791 & 4512 & 2559 \\
\hline Trivium_x $8 f p$ & 7712.91 & 873 & 4737 & 2976 \\
\hline Trivium_x16 & 7425.73 & 855 & 4410 & 3016 \\
\hline Trivium_x16mp & 7637.41 & 979 & 4494 & 3143 \\
\hline Trivium_x16fp & 8992.87 & 1147 & 5063 & 3929 \\
\hline
\end{tabular}

The FPLP Trivium ciphers have more logical cells and more combinational area than the other Trivium cipher proposals. The non-combinational area is similar in all versions except for the 16-bit FPLP version (9\% larger), due to the increase in the number of flip flops in some odd and even parallel registers. Of particular interest are the increases in the combinational cell area shown in the eight and sixteen bit FPLP versions ( $41 \%$ and $86 \%$ respectively). These are due to the large number of bits that have to be feedback, which raises the number of combinational cells considerably.
The Synopsys synthesis of the MPLP Triviums, the version that evolved best with the number of output bits, generate designs with fewer combinational cells than the FPLP versions. However, its area in multi-bit cases ("x2", "x8" or " $\mathrm{x} 16$ ") increased more than in the standard Trivium version.

TABLE II

PERCENTAGES OF AREA AND NUMBER OF LOGICAL CELLS, WITH REFERENCE

\begin{tabular}{|c|c|c|c|c|}
\hline \multirow[b]{2}{*}{ Trivium } & \multicolumn{4}{|c|}{ Library: TSMC $90 \mathrm{~nm}$} \\
\hline & $\begin{array}{l}\text { Total } \\
\text { Area }\end{array}$ & $\begin{array}{c}\text { Logic } \\
\text { Cells }\end{array}$ & $\begin{array}{c}\text { Area Non } \\
\text { Comb. Cells }\end{array}$ & $\begin{array}{c}\text { Area } \\
\text { Comb. Cells }\end{array}$ \\
\hline Trivium_x1 & - & - & - & - \\
\hline Trivium_xlmp & - & - & - & - \\
\hline Trivium_xlfp & - & - & - & - \\
\hline Trivium_x2 & $1 \%$ & $1 \%$ & $0 \%$ & $4 \%$ \\
\hline Trivium_x $2 m p$ & $1 \%$ & $-3 \%$ & $2 \%$ & $-1 \%$ \\
\hline Trivium_x $2 f p$ & $2 \%$ & $6 \%$ & $0 \%$ & $7 \%$ \\
\hline Trivium_x8 & $8 \%$ & $16 \%$ & $-1 \%$ & $26 \%$ \\
\hline Trivium_x8mp & $7 \%$ & $19 \%$ & $1 \%$ & $20 \%$ \\
\hline Trivium_x $8 f p$ & $14 \%$ & $41 \%$ & $2 \%$ & $41 \%$ \\
\hline Trivium_x16 & $17 \%$ & $37 \%$ & $0 \%$ & $53 \%$ \\
\hline Trivium_x16mp & $16 \%$ & $47 \%$ & $0 \%$ & $48 \%$ \\
\hline Trivium_x16fp & $33 \%$ & $85 \%$ & $9 \%$ & $86 \%$ \\
\hline
\end{tabular}

$\left.* \%=\%\left(T_{\text {standard }}-T_{F P L P-M P L P}\right) / T_{\text {standard }}\right)$.

As it is summarize in Table II, the MPLP and FPLP Trivium proposals maintain a good relationship in terms of their numbers of cells compared to the standard version in the 
radix-1 and radix-2 implementations. Their areas are very similar when implementations with the same radix are compared, although at radix- 8 and radix-16 the difference between them becomes greater, especially in the radix-16 FPLP version, where there is a clear difference in resources and area.

The differences between the standard and low power versions in terms of total area are also shown graphically in Fig. 5, with the same conclusions. The FPLP versions have always more area than the others and for radix- 8 and radix- 16 the difference is greater.

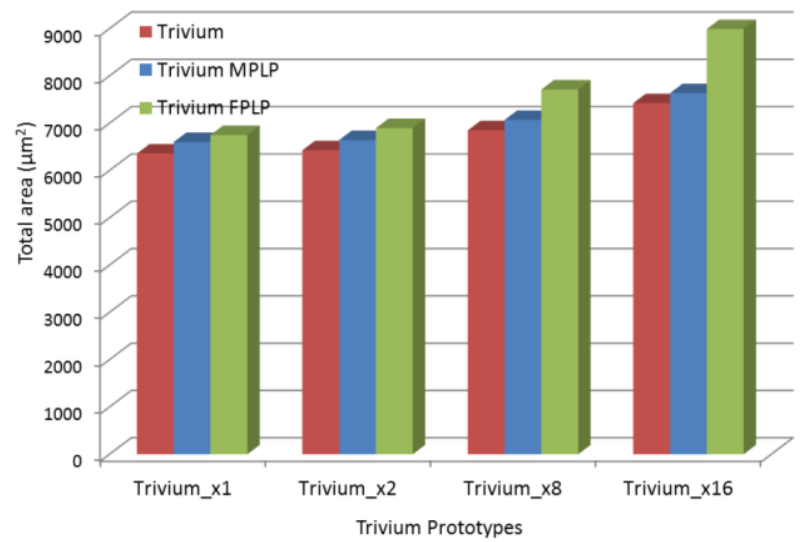

Fig. 5. Multi-Radix Trivium area report.

\section{B. Power consumption implementation report}

Power reports provided by the Encounter RTL-GDSII tool for the multi-radix standard and MPLP versions of Trivium are shown in Table III. All the power results were obtained including activity files obtained from post place-and-route simulations with full timing and parasitic parameters. We used the worst-case corner for the simulations.

TABLE III

MULTI-RADIX TRIVIUM POST-LAYOUT REPORT. $\mathrm{VDD}=1,08 \mathrm{~V}-\mathrm{T}=125^{\circ} \mathrm{C}-50 \mathrm{MHz}$-worst case timing

\begin{tabular}{|c|c|c|}
\hline \multirow[b]{2}{*}{ Trivium } & \multicolumn{2}{|c|}{ Dynamic power consumption } \\
\hline & $\mu W$ & $\% *$ \\
\hline Trivium_x1 & 330,6 & - \\
\hline Trivium_xImp & 227,0 & 31,3 \\
\hline Trivium_xlfp & 179,8 & 45,6 \\
\hline Trivium_x2 & 339,5 & - \\
\hline Trivium_x2mp & 231,7 & 31,8 \\
\hline Trivium_ $x 2 f p$ & 197,4 & 41,9 \\
\hline Trivium_x8 & 395,6 & - \\
\hline Trivium_x8mp & 330,1 & 16,6 \\
\hline Trivium_x $8 f p$ & 334,8 & 15,4 \\
\hline Trivium_x16 & 418,5 & - \\
\hline Trivium_x16mp & 423,7 & $-1,2$ \\
\hline Trivium_xl6fp & 512,18 & $-22,4$ \\
\hline
\end{tabular}

When the power consumption of the three Trivium implementations was compared, it was noticed that the low power implementations have lower dynamic power consumption than the standard Trivium up to radix-16, where there is no improvement due to the increase in the number of internal net connections and, for the MPLP version, the progressive decrease in bit numbers in the parallelized registers: from 196 bits in radix-1 and radix-2 to 144 and 96 bits in radix-8 and radix-16, respectively.

As was expected, power consumption was found to increase with the level of parallelization. However, the Trivium versions proposed to reduce power consumption proved effective up until radix-8 (although they were unsuccessful for radix-16). The MPLP and FPLP proposals showed significant improvements of about $31-45 \%$ for radix-1 and radix-2 versions and a significant improvement (15-16\%) in radix-8 version. The FPLP radix- 1 and radix- 2 versions offered the best power reduction: more than $45 \%$ less than the standard version. The dynamic power differences between the standard and low power versions are also shown graphically in Fig. 6, with the same conclusions.

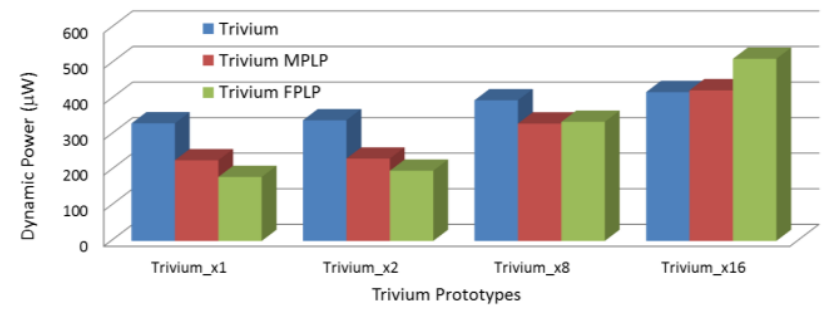

Fig. 6. Multi-Radix Trivium power report.

The FPLP version can be seen to improve the power consumption of all the standard versions of Trivium up to the radix-16 version, where there is no improvement. It is important to note that, in the radix-1, the FPLP version almost halves the consumption of the standard Trivium. In fact, in comparison with the MPLP version, FPLP has much better values than MPLP for the radix-1 and radix- 2 versions. For radix- 8 , both are very similar.

Finally, Fig. 7 shows a bar diagram with the percentages of improvement in power consumption. The percentages are positive when power consumption is lower than in the standard version and negative when it is not. Obviously, the improvement value for the standard version itself is 0 . This figure clearly illustrates the lower power consumption of the MPLP and FPLP versions, except in the case of 16 bits, where they consume more.

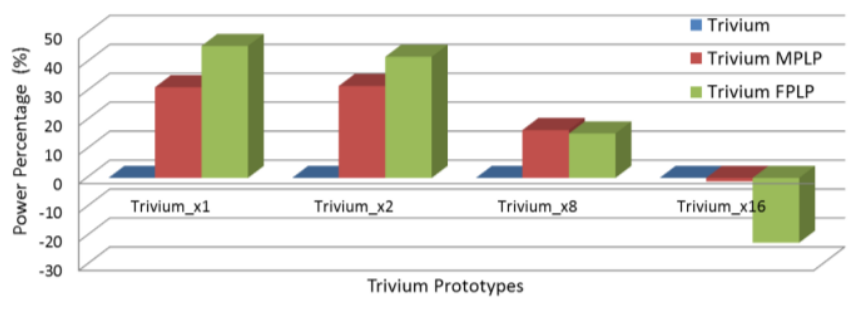

Fig. 7. Multi-Radix Trivium power percentage. 


\section{Multi-RADiX TRIVIUM Results COMPARISON}

When the power consumption of the three Trivium implementations is compared, it can be seen that the FPLP implementation offers the best results in power reduction for radix-1 and radix-2 (see Table III and Fig. 7). The power consumption of the MPLP Trivium is lower than that of the standard implementation but slightly worse than that of the FPLP version.

The disadvantage of FPLP is that it has more cell area than MPLP, although the slight overhead in area is less than $7 \%$ in radix- 1 and radix-2 and $21 \%$ in radix- 16 .

The MPLP version has no cell area penalties in comparison with the standard version (3-4\%, as shown in Table I), and is therefore still a good option for radix-1 and radix-2.

The results clearly show that the reduction in dynamic power consumption in the FPLP Trivium implementation is best in radix-1 and radix-2 (about 45\%), while the MPLP implementation offers a power reduction of about $31 \%$ for the same radix. However for radix- 8 , the power consumption of both low power versions is very similar.

\section{CONCLUSION}

In this paper, we have proposed new low power multi-radix Trivium designs: MPLP and FPLP. Both are based on logic parallelization techniques. Twelve versions of Trivium (radix$1,-2,-8$ and -16 for each standard, MPLP and FPLP proposal) were designed using semicustom design methodologies in 90 $\mathrm{nm}$ technology. All the versions of Trivium were characterized for area and dynamic power consumption with post-synthesis and post-layout data.

In the cases of radix- 1 and radix- 2 bits, the two versions implemented, MPLP and FPLP, greatly reduced the power consumption of the standard version of the Trivium, while, for radix-8, the reduction was significant but considerably lower.

The FPLP version raised the power consumption improvement percentage achieved by MPLP by between 10 and 15 points for radix-1 and radix-2. For radix-8, the reduction achieved by both versions was similar. In the case of radix-16, neither of the low power versions reduced power consumption.

The area penalty and cell numbers obtained with this technique were very low (less than 13\% for FPLP radix-8), while the improvement in dynamic power consumption was quite high (more than 15\% for FPLP radix-8).

The FPLP Trivium version offers greater power reduction than the MPLP Trivium, but also produces a slight increase in the complexity of the implementation and the logic used, which in turn increases the final area.

Hence, MPLP and FPLP implementations are presented as very competitive options for incorporating hardware security solutions for very low power Internet of Things (IoT) applications.

\section{REFERENCES}

[1]: Manifavas, G. Hatzivasilis, K. Fysarakis and Y. Papaefstathiou, "Lightweight Cryptography for Embedded Systems - A Comparative Analysis," in Security and Communication Networks, vol. 9, no. 10, p.
1226-1246, 2015. DOI: 10.1007/978-3-642-04101-3.

[2]: T. Eisenbarth, . S. Kumar, C. Paar, A. Poschmann y L. Uhsadel, "A survey of lightweight-cryptography implementations," in IEEE Design \& Test of Computers, vol. 24, $\mathrm{n}^{\circ}$ 6, pp. 522-533, 2007.

[3]: Bui, D. H., Puschini, D., Bacles-Min, S., Beigne, E., \& Tran, X. T, "Ultra Low-Power and Low-Energy 32-bit Datapath AES Architecture for IoT Applications," in IEEE International Conference on Integrated Circuit Design and Technology, 27-29 June 2016. DOI: 10.1109/ICICDT.2016.7542076.

[4]: B. Preneel, C. Paar y J. Pelzl, Understanding cryptography: a textbook for students and practitioners. Springer-Verlag Berlin Heidelberg 2010. DOI: 10.1007/978-3-642-04101-3.

[5]: eSTREAM: the ECRYPT Stream Cipher Project. Available: http://www.ecrypt.eu.org/stream/

[6]: Matthew Robshaw Olivier Billet (Eds.). New Stream Cipher Designs. The eSTREAM Finalists, Springer 2008. DOI: 10.1007/978-3-54068351-3.

[7]: Kocheta, M. ; Sujatha, N. ; Sivakanya, K. ; Srikanth, R. ; Shetty, Sridhar ; Ananda Mohan, P.V. "A review of some recent stream ciphers," in International conference on Circuits, Controls and Communications (CCUBE), 2013 , Page(s): 1- 6.

[8]: Parrilla, L. ; Castillo, E. ; Morales, D.P. ; Garcia, A. ; Molina, F.S. ; Florido, J. "Compact cryptoprocessor for securing wireless communications in FECG portable instrumentation," in Conference on Design of Circuits and Integrated Circuits (DCIS), 2014, Page(s): 1-6. DOI: 10.1109/DCIS.2014.7035574

[9]: Jiezhong Gong ; Gongliang Chen ; Linsen Li ; Jianhua Li. "A secure authentication protocol for RFID based on Trivium," in International Conference on Computer Science and Service System (CSSS), 2011 , Page(s): 107- 109. DOI: 10.1109/CSSS.2011.5974817

[10]: Marmolejo-Tejada, J.M. ; Trujillo-Olaya, V. ; Velasco-Medina, J. "Hardware implementation of Grain-128, Mickey-128, Decim-128 and Trivium," in ANDESCON, 2010 IEEE, Page(s): 1- 6. DOI: 10.1109/ANDESCON.2010.5632901.

[11]: Feldhofer, M.: "Comparison of low-power implementations of Trivium and Grain," in State of the Art of Stream Ciphers Workshop (SASC 2007)".

[12]: Good, T., Benaissa, M.: "Hardware results for selected stream cipher candidates," in State of the Art of Stream Ciphers Workshop (SASC 2007), eSTREAM, ECRYPT Stream Cipher Project, Report 2007/023 (2007).

[13]: T. Schneider et al., "Low-Voltage Low-Power Parallelized Logic Modules," in Proc. PATMOS95, Paper S4.2, Oldenburg. October 4-6, 1995, Germany.

[14]: A. P. Chandrakasan, R. W. Broderesen, Low Power digital CMOS design, Kluwer Academics Publishers, Boston, 1995. DOI:10.1007/9781-4615-2325-3.

[15]: B. Lin, H. de Man. "Low-power driven technology mapping under timing constraints," in Proc. ICCAD, 1993, pp 421-427.

[16]: J. M. Mora-Gutiérrez, C. J. Jiménez-Fernández, M. Valencia-Barrero: "Trivium hardware implementations for power reduction," in International Journal of Circuit Theory and Applications. Published online in Wiley Online Library (wileyonlinelibrary.com) Nov. 2016. DOI: $10.1002 /$ cta.2281.

[17]: C. Piguet, J. M.. Masgonty, V. von kaenel, T. Schneider, "Logic design for low-voltage/low-power CMOS circuits," in. Int. Symp. on low-Power Design, Dana Point, CA, Apr 23-26, 1995, pp. 117-122

[18]: C. Piquet, Low-Power CMOS Circuits technology, Logic Design and CAD Tools. CRC Press, 2006. DOI: 10.1201/9781420036503.

[19]: C. De Canniere y B. Preneel, "Trivium, A Stream Cipher Construction Inspired by Block Cipher Design Principles," in State of the Art of Stream Ciphers Workshop (SASC 2006), eSTREAM, ECRYPT Stream Cipher Project, Report 2006/021. (2006). DOI:10.1007/11836810_13.

[20]: R. Chadha y J. Bhasker, An ASIC Low Power Primer; Analysis, Techniques and Specification. Springer, 2013. DOI:10.1007/978-14614-4271-4.

[21]: P. R. Panda, B. V. N. Silpa, A. Shrivastava y K. Gummidipudi, Powerefficient system design, Springer, 2010. DOI: 978-1-4419-6387-1. 


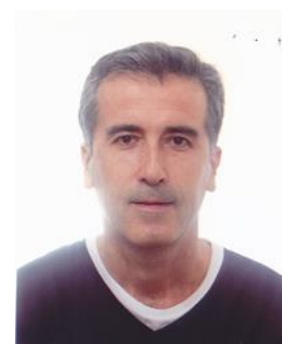

José Miguel Mora received the BSc. degree and the M.Sc. degree in telecommunication engineering, specializing in microelectronics, from the Technical University of Madrid, Madrid, Spain, in 1990 and 1992, respectively. Since April 1992, he is a Member of the Technical Staff at the Instituto de Microelectrónica de Sevilla (IMSECNM, CSIC/University of Seville), where he is currently an Assistant Manager of the Research and Development section. His main areas of interest include the design and testing digital and mixed integrated circuits and field-programmable gate arrays, and design and test of IC for cryptography. He has been in the design teams of different research projects dealing with integrated circuits and systems.

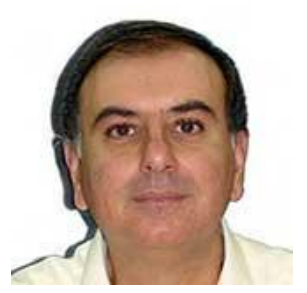

Carlos J. Jiménez. Carlos J. Jiménez received the B.Sc. and Ph.D. degrees in physics from the University of Seville, Seville, Spain, in 1989 and 2000, respectively. From 1990 to 2001, he was been with the Instituto de Microelectrónica de Sevilla (IMSECNM, CSIC/University of Seville). Since 2001, he is Associate Professor with the Department of Electronic Technology, University of Seville. His current research interests include the design and test of IC for cryptography, hardware implementations of ciphers for IoT applications, secure communications, and design methodologies and CAD tools. He is co-author of two books and more than 50 scientific papers, and has collaborated in more than 20 research projects (national and international).

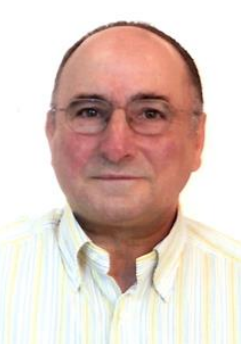

Manuel Valencia. received the B.Sc. degree in 1976, the M.Sc. degree in 1977, and the Ph.D. degree in 1986 in Electronic Physics from the University of Seville, Spain. He is currently with the Institute of Microelectronics of Seville, IMSE-CNM-CSIC and also with the Department of Electronic Technology of the University of Seville, where he has been a Full Professor since 2000. His current research interests are in the areas of CMOS Digital VLSI Design, low-power and low-noise CMOS, (lightweight) cryptographic circuits, computer arithmetic, processor architectures, timing in VLSI digital, and modelling and simulation at the logic-timing level. 